\title{
Torsional Buckling and Writhing Dynamics of Elastic Cables and DNA
}

S. Goyal, N. C. Perkins, C. L. Lee

This article was submitted to American Society of Mechanical Engineers $19^{\text {th }}$ Biennial Conference of Mechanical Noise and Vibration, Chicago, IL - September 2 - 6, 2003

\section{February 14, 2003}




\section{DISCLAIMER}

This document was prepared as an account of work sponsored by an agency of the United States Government. Neither the United States Government nor the University of California nor any of their employees, makes any warranty, express or implied, or assumes any legal liability or responsibility for the accuracy, completeness, or usefulness of any information, apparatus, product, or process disclosed, or represents that its use would not infringe privately owned rights. Reference herein to any specific commercial product, process, or service by trade name, trademark, manufacturer, or otherwise, does not necessarily constitute or imply its endorsement, recommendation, or favoring by the United States Government or the University of California. The views and opinions of authors expressed herein do not necessarily state or reflect those of the United States Government or the University of California, and shall not be used for advertising or product endorsement purposes.

This is a preprint of a paper intended for publication in a journal or proceedings. Since changes may be made before publication, this preprint is made available with the understanding that it will not be cited or reproduced without the permission of the author. 


\title{
TORSIONAL BUCKLING AND WRITHING DYNAMICS OF ELASTIC CABLES AND DNA
}

\author{
S. Goyal \\ Graduate Student, Department of Mechanical Engineering, The University of Michigan, Ml-48105-2125, \\ USA \\ N. C. Perkins \\ Fellow, ASME \\ Professor, Department of Mechanical Engineering, The University of Michigan, MI-48105-2125, USA \\ Christopher L. Lee \\ Member, ASME \\ New Technologies Engineering Division, Lawrence Livermore National Laboratory, 7000 East Ave., \\ Livermore, CA 94550, USA
}

\begin{abstract}
Marine cables under low tension and torsion on the sea floor can undergo a dynamic buckling process during which torsional strain energy is converted to bending strain energy. The resulting threedimensional cable geometries can be highly contorted and include loops and tangles. Similar geometries are known to exist for supercoiled DNA and these also arise from the conversion of torsional strain energy to bending strain energy or, kinematically, a conversion of twist to writhe. A dynamic form of Kirchhoff rod theory is presented herein that captures these nonlinear dynamic processes. The resulting theory is discretized using the generalizedmethod for finite differencing in both space and time. The important kinematics of cross-section rotation are described using an incremental rotation "vector" as opposed to traditional Euler angles or Euler parameters. Numerical solutions are presented for an example system of a cable subjected to increasing twist at one end. The solutions show the dynamic evolution of the cable from an initially straight element, through a buckled element in the approximate form of a helix, and through the dynamic collapse of this helix through a looped form
\end{abstract}

\section{INTRODUCTION}

Cables laid upon the sea floor may form loops and tangles as illustrated in Fig. 1. The loops (sometimes also referred to as hockles) may cause localized damage and, in the case of fiber optic cables, may also prevent signal transmission. These highly nonlinear deformations are initiated by conditions of low cable tension (or slight compression) and torsion sufficient to induce a torsional "buckling" of the cable. Several prior investigations of cable loop formation have employed nonlinear equilibrium (static) rod theories to analyze the equilibrium forms of cables under torsion and low tension; see, for example, Coyne [1], Rosenthal [2, 3], Liu [4], Tan and Witz [5]. The stability of these equilibrium forms may be assessed using local stability analyses as in $\mathrm{Lu}$ and Perkins $[6,7]$

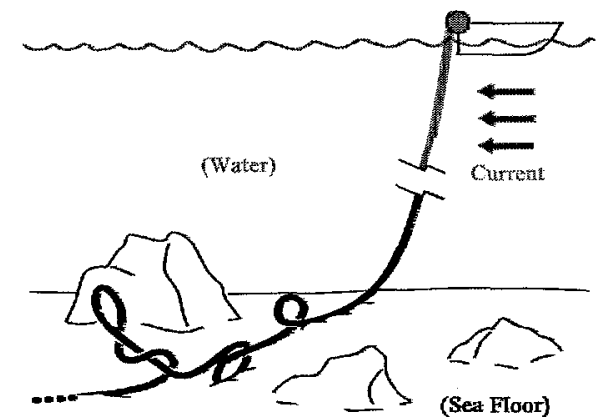

Figure 1: Low tension cable forming loops and tangles on the sea floor.

The overail buckling process, however, is inherently a dynamic process, and this fact has recently been recognized by Gatti-Bono and Perkins [8] who employ a nonlinear dynamic rod theory to simulate loop formation under cable compression.

It is interesting to observe that the looped and tangled forms of marine cables are topologically similar to the supercoiled states of DNA [9]. For instance, the tangle depicted in Fig. 1 for a marine cable resembles the plectonemic structures (illustrated in Fig. 2) that form in stranded and looped DNA following conversions of twist to writhe. The geometry of DNA has a controlling influence on its biological functions including the processes of transcription and replication [9]. The equilibrium (static) rod theories utilized for cable looping have (with modifications) also been employed to study (the long-length scale) supercoiling of DNA; see, for example, [10-17]. Like the marine cables above, the transitions of DNA between supercoiled states is inherently a dynamical process, yet little is actually known about it. 


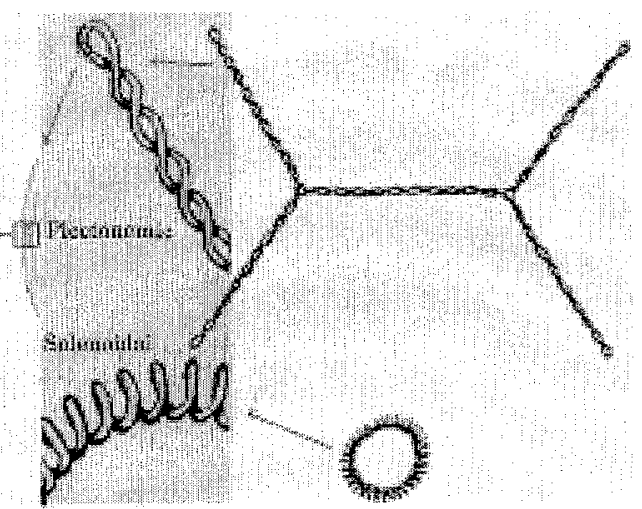

Figure 2: DNA resembles a helical ladder (far left) that, when viewed over long length-scales, has been modeled as a rod. The entire molecule may supercoil, and the supercoiling may be plectonemic or solenoidal as illustrated. (Illustrations courtesy of [29]).

The objective of this study is to provide a rod theory for cables that can be used to capture dynamic buckling under increasing torsion (twist). The dynamics of this process as well as the model and numerical methods described herein, may also be a useful starting point for understanding the writhing dynamics of DNA and dynamic transitions between supercoiled states.

We open by summarizing a nonlinear dynamic rod model used by Gatti-Bono and Perkins [18] to describe highly contorted states of elastic cables. Next, this model is discretized by finite differencing following Gatti-Bono and Perkins [18] as well as Gobat and Grosenbaugh [19] who employ the generalized- method by Chung and Hulbert [20] for integration with respect to time. The resulting numerical model is exercised on a prototypical example of a cable element subjected to increasing twist. We close with a summary of our conclusions.

\section{PHYSICAL MODEL}

Consider the infinitesimal element of a cable [18] in Fig. 3. Let the vector triad $\left\{e_{i}\right\}$ define an inertial reference frame and the vector triad $\left\{a_{i}\right\}$ define a local reference frame fixed to the cross-section and aligned with the tangent and the "principal torsion-flexure axes" [21]. The quantities $Q$ and $B$ denote the external torque and force per unit length, respectively, while $q$ and $f$ denote the internal moment and force, respectively, that act on the cross-section (internal stress resultants).

At each spatial point on the cable centerline is defined by the Lagrangian variable $s$. Four vectors are required to define the dynamic state of the cable cross-section and the internal stress resultants. These include: the linear velocity $v$ of the cross-section at the centerline, the angular velocity $\omega$ of the cross-section, the curvature (or Darboux vector) $\kappa$ of the centerline at the crosssection, and the internal force $f$. We express all quantities and derivatives in the local frame, unless otherwise stated.

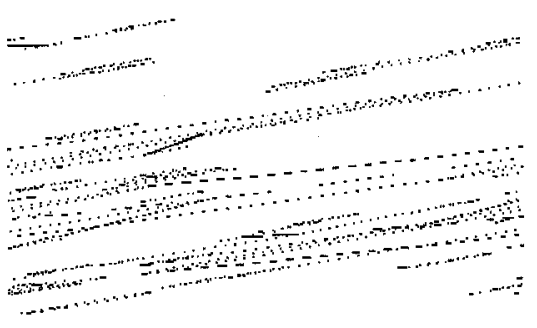

Figure 3: Infinitesimal element of Kirchhoff rod for formulating equations of motion.

The kinematical quantities $\omega$ and $x$ are smooth and they are related by the requirement that the order of spatial and temporal differentiation are interchangeable ${ }^{1}$ :

$$
\frac{\partial \omega}{\partial s}+\kappa \times \omega=\frac{\partial \kappa}{\partial t}
$$

We assume that the centerline is inextensible, which leads to the requirement that

$$
\frac{\partial v}{\partial s}+\kappa \times v=\omega \times a_{3}
$$

The balance law for linear momentum of the element in Fig. 3 is

$$
\frac{\partial f}{\partial s}+\kappa \times f=\rho_{c} A_{c}\left\{\frac{\partial v}{\partial t}+\omega \times v\right\}-B
$$

and that for angular momentum is

$$
\frac{\partial q}{\partial s}+\kappa \times q=I \frac{\partial \omega}{\partial t}+\omega \times(I \omega)+f \times a_{3}-Q
$$

Here, $B$ and $Q$ represent any distributed force and moment, respectively, produced by the surrounding environment including gravity, buoyancy, hydrodynamic drag and added mass, contact, etc. In general, these quantities are nonlinear functions of the state vectors. $I$ is the moment of inertia tensor for the cable cross-section about the triad $\left\{a_{i}\right\}$.

The internal moment is related to the curvature and angular velocity through an assumed (linear) constitutive law that includes the influence of intrinsic curvature/ torsion, $\kappa_{0}$, and structural damping, $C$ :

$$
q=\left[\begin{array}{ccc}
E J_{1} & 0 & 0 \\
0 & E J_{2} & 0 \\
0 & 0 & G J_{3}
\end{array}\right]\left(x-\kappa_{0}\right)+\left[\begin{array}{ccc}
C_{1} & 0 & 0 \\
0 & C_{2} & 0 \\
0 & 0 & C_{3}
\end{array}\right] \omega
$$

\footnotetext{
${ }^{1}$ A derivation of this result is found in Section 4 following Eq. (25).
} 
The material and geometric parameters used in Eq. (5) are defined in Tables 1 and 2. Though internal structural damping might also be modeled as being proportional to $\left(\frac{\partial x}{\partial t}\right)_{\left\{e_{i}\right\}}$, relating it to $\omega$ here offers simplicity at the expense of additional damping for rigid-body rotations. Alternative formulations of damping could be incorporafed at this stage.

The constitutive law Eq. (5) is substituted into the angular momentum balance law, Eq. (4). The resulting four field equations (Eq. (4) substituted with Eq. (5), and Eqs. (1)-(3)) constitute a set of four (vector) equations in the four unknowns represented by the state vector:

$$
Y(s, t)=\{v, \omega, \kappa, f\}
$$

We consolidate the field equations in the form

$$
M(Y, s, t) \frac{\partial Y}{\partial t}+K(Y, s, t) \frac{\partial Y}{\partial s}+F(Y, s, t)=0
$$

Observe that $M$ is always a singular matrix (no time derivative appears in the constraint equation $\mathrm{Eq}$. (2). K is always non-singular (but for a very flexible cable, it may become ill-conditioned).

For a three-dimensional configuration, the dimension of $Y$ is 12. In order to solve the set of first-order partial differential equations Eq. (7), we must also specify

1. $Y(s, 0)$ as the initial conditions (initial configuration of the cable and its initial velocity and angular velocity), and

2. six components of $Y(0, t)$ with six of $Y(L, t)$ as the boundary conditions. In general, the boundary conditions may be implicit and nonlinear, e.g. $\Phi\left(Y, \frac{\partial Y}{\partial t}, t\right)=0$, which would then require numerical solution together with the partial differential equations, Eq. (7).

\section{NUMERICAL SOLUTION ALGORITHM}

Following the work of Gobat and Grosenbaugh [19], we discretize by finite differencing and make reference to the space-time discretization grid shown in Fig. 4. We denote spatial derivatives by a superscript prime and temporal derivatives by a superscript dot.

Starting with initial conditions, and for each successive time step, we integrate along $s$ and use the shooting method to satisfy all boundary conditions at the two ends. Thus, to solve for $Y$ at the open node $(i, j)$ in Fig. 4, we use the known solution $Y$ at the two shaded nodes $(i-1, j)$ and $(i-1, j-1)$ known from the prior time step, as well as to the solution $Y$ at the partially shaded-circle node (current time step, prior spatial step, $(i, j-1)$ as described next. All (spatial and temporal) derivatives are formed using the Generalized$\alpha$ method described below.

We begin with $Y$ and $Y^{\prime}$ known from the initial conditions $(t=0)$, and then compute the initial value of $\dot{Y}$ from the governing equations, Eq. (7). Though $M$ is singular, to evaluate $\dot{Y}$, we simplify by choosing $\dot{f}=0$ to start with, as there is no dependence on $\dot{f}$ in Eq. (7). Also, any initial condition must satisfy

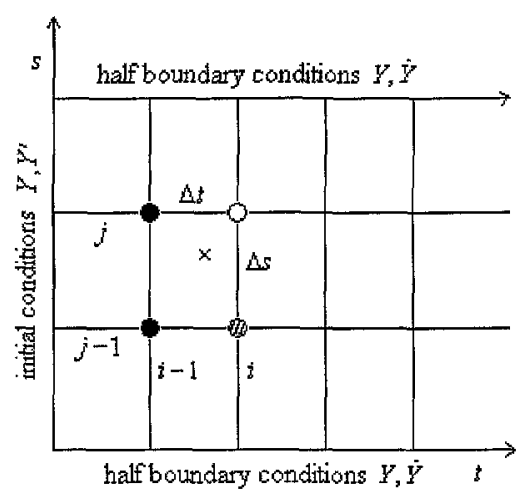

Figure 4: Space-time discretization grid.

$$
\frac{\partial \dot{v}}{\partial s}+\dot{\kappa} \times v+\kappa \times \dot{v}=\dot{\omega} \times a_{3}
$$

which is the time-derivative of the inextensibility constraint, Eq. (2).

Finite differencing of $\mathrm{Eq}$. (7) in time is achieved using the Generalized- method, whose advantages in this application are discussed in Gobat et al. [22],

$$
\begin{gathered}
M^{1-\alpha_{i}} \dot{Y}^{1-\alpha_{i}}+K^{1-\beta_{t}} Y^{\prime 1-\beta_{i}}+F^{1-\beta_{t}}=0 \\
()^{1-x}=(1-x) O_{i}+x()_{i-1}
\end{gathered}
$$

Here $\alpha_{t}$ is introduced as a "mass-averaging" numerical parameter while $\beta_{z}$ is a "stiffness-averaging" numerical parameter. The subscript $t$ indicate that the averaging is done with respect to time as explained in Eq. (10) for $x=$ either $\alpha_{t}$ or $\beta_{t}$. Note however, that for many applications, $M$ and $K$ are constant and hence they do not require averaging in the difference equation. We assume this is the case for simplicity. ${ }^{2}$

The solution is known at the previous time step $(i-1)$ (see shaded nodes in Fig. 4) and we move these terms to the right-hand side of Eq. (9) in creating the known nonhomogeneous term $H$. Subsequent finite differencing in space yields,

$$
\left(1-\alpha_{t}\right) M \dot{Y}_{i}^{1-\alpha_{s}}+\left(1-\beta_{t}\right)\left(K Y_{i}^{\prime 1-\beta_{s}}+F_{i}^{1-\beta_{s}}\right)=H
$$

where the right-hand side

$$
H=\alpha_{t} M \dot{Y}_{i-1}^{1-\alpha_{s}}+\beta_{t}\left(K Y_{i-1}^{\prime 1-\beta_{s}}+F_{i-1}^{1-\beta_{s}}\right)
$$

is known, and where

$$
0^{1-x}=(1-x) 0_{j}+x()_{j-1}
$$


For the temporal and spatial derivatives, we employ the Newmark-like formulations,

$$
\begin{gathered}
\dot{Y}_{i}=\frac{Y_{i}-Y_{i-1}}{\gamma_{t} \Delta t}-\frac{1-\gamma_{t}}{\gamma_{t}} \dot{Y}_{i-1} \\
Y_{j}^{\prime}=\frac{Y_{j}-Y_{j-1}}{\gamma_{s} \Delta s}-\frac{1-\gamma_{s}}{\gamma_{s}} Y_{j-1}^{\prime}
\end{gathered}
$$

The Newmark constants $\gamma_{t}$ and $\gamma_{s}$ are numerical parameters that control the averaging of time and space derivatives. A Newmark-like method for time integration was used by Sun [23] for cable dynamics simulations. In the Generalized- method, the numerical parameters $\left(\left\{\alpha_{t}, \beta_{t}, \gamma_{t}\right\}\right.$ or $\left.\left\{\alpha_{s}, \beta_{s}, \gamma_{s}\right\}\right)$ are selected to satisfy optimal numerical accuracy and stability as described briefly below.

Upon substituting Eq. (14) and Eq. (15) into Eq.(11), and consolidating all nonhomogeneous terms into $H$, we arrive at the algebraic equations

$$
\hat{K} Y_{i, j-1}^{\prime}+A\left(Y_{i, j}\right)=B\left(Y_{i, j-1}\right)+H
$$

that are linear in $Y^{\prime}$ and nonlinear in $Y$. From here forward, we drop the subscript $i$ for notational simplicity. Starting from a guessed solution $Y^{*}$, we form $\dot{Y}^{*}$ from the Newmark algorithm Eq. (14) and then form $Y^{\prime *}$ using the governing equations Eq. (7). Here, the superscript * will indicate a quantity that depends on the guessed solution and that is also updated as the algorithm proceeds. Linearizing $A$ and $B$ about the guessed solution $Y^{*}$ leads to the approximation to $\mathrm{Eq}$. (16).

$$
\hat{K}\left(Y_{j-1}^{\prime}\right)^{*}+R^{*}+A_{y}^{*} Y_{j}=S^{*}+B_{y}^{*} Y_{j-1}+H
$$

which further reduces to

$$
A_{y}^{*} Y_{j}=B_{y}^{*} Y_{j-1}+H^{*}
$$

after consolidating all nonhomogeneous terms in $H^{*}$.

This linear nonhomogeneous algebraic equation is now employed to integrate over space. Starting from $s=0$, we apply the shooting method as discussed in Gatti-Bono and Perkins [18]. In short, we assume that $Y$ at any $s$ belongs to an affine solution space. With the known boundary conditions at $s=0$, we find a basis of the solution space at $s=0$. Then, we determine how the solution space (the chosen basis) transforms through to other end according to Eq. (18). Finally, we fix the linear combination of the basis vectors to satisfy the terminal boundary conditions in arriving at an updated solution at all $s$ as our next guess. We then update the coefficient matrices in Eq. (18) with the next guess and iterate this cycle until convergence is achieved.

To select the numerical parameters, we draw an analogy to the scalar problem studied in Gobat and Grosenbaugh [19]. We can choose $\left(\alpha_{t}, \beta_{t}, \gamma_{t}\right)=\left(\alpha_{s}, \beta_{s}, \gamma_{s}\right)=(\alpha, \beta, \gamma)$ to achieve the same accuracy and stability in space and time. Second-order accuracy on truncation error requires that the numerical parameters satisfy

$$
\gamma+\alpha-\beta=\frac{1}{2}
$$

Stability requires that the amplification matrix in recursion have a spectral radius (magnitude of the largest eigenvalue) less than unity. Unconditional stability requires

$$
\alpha \leq \frac{1}{2}, \beta \leq \frac{1}{2}, \gamma \geq \frac{1}{2}
$$

The spectral radius is a measure of numerical dissipation. We want to preferentially dissipate high frequency components of the response, low frequency components are important and should have minimal dissipation. Chung and Hulbert [20] achieved such controllable dissipation by imposing smoothness requirements on the spectral radius. Their requirement when combined with Eq. (19) and Eq. (20) enables one [19] to relate all three numerical parameters to a single parameter, $\lambda_{\infty}$, where $\left|\lambda_{\infty}\right|$ is the minimum spectral radius (or, equivalently, the maximum numerical dissipation).

$$
\alpha=\frac{3 \lambda_{\infty}+1}{2 \lambda_{\infty}-2}, \beta=\frac{\lambda_{\infty}}{\lambda_{\infty}-1}
$$

\section{KINEMATICS OF CROSS-SECTION ROTATION}

The solution algorithm reviewed above provides the solution $Y(s, t)=\{v, \omega, \kappa, f\}$ as time marches forward. If any external forces and/or moments $(B$ and $Q$ ) are specified in the inertial frame (e.g., weight and buoyancy), then these quantities need to resolved into components along the local frame as the solution proceeds. Doing so requires knowledge of the transformation $L$ from the inertial frame to the local frame.

$$
\left\{e_{j}\right\}=\left\{a_{i}\right\} L
$$

Likewise, specific boundary conditions may require use of this transformation during solution. In addition, we need this transformation in post-processing the solutions for the curvature vector $\kappa$ in arriving at the three-dimensional space curve that describes the cable centerline.

Differentiating Eq. (22) with respect to time leads to

$$
\frac{\partial L}{\partial t}=-\tilde{\omega} L
$$

In deriving $\mathrm{Eq}$. (23) above, we used the cross-product formula $\omega \times a_{i}=\tilde{\omega} a_{i}$, where $\tilde{\omega}$ is the skew-symmetric form of the vector $\omega$,

$$
\tilde{\omega}=\left[\begin{array}{ccc}
0 & -\omega_{3} & \omega_{2} \\
\omega_{3} & 0 & -\omega_{1} \\
-\omega_{2} & \omega_{1} & 0
\end{array}\right]
$$

We can now solve Eq. (23) for $L$ at each time step from known solutions of $\omega$.

Equivalently, for specific boundary conditions, we might know $L$ at one end for all time and then use the computed curvature $\kappa$ to determine the transformation along the remainder of the cable per 


$$
\frac{\partial L}{\partial s}=-\tilde{\kappa} L
$$

We use Eqs. (23) and (25) to deduce ${ }^{3}$ the constraint Eq. (2).

We update the transformation $L$ using the decomposition

$$
L_{i}=\exp (-\tilde{\theta}) L_{i-1}
$$

where we use the fact that any orthogonal transformation can be represented by exponentiation of a skew-symmetric matrix. The quantity $\theta$ has a simple physical interpretation. The transformation of the local frame $\left\{a_{i}\right\}$ in one time step (say from $i-1$ to $i$ ), can be accomplished by a single rotation $\|\theta\|_{2}$ about a unit vector $u$, where $\|\left.\theta\right|_{2}$ denotes the $L_{2}$ norm of $\theta$. If we decompose $u$ in the lacal frame $\left\{a_{i}\right\}$, then the resulting vector $\|\theta\|_{2} u$ is exactly the incremental rotation vector $\theta$ whose skew-symmetric form $\tilde{\theta}$ satisfy Eq. (26). We note that $\theta \approx \int_{i-1}^{i} a d t$ with the approximation becoming exact for infinitesimal rotations (i.e., smaller time steps) After estimating $\theta$ from $\omega$, we can use the rotation formula ${ }^{4}$ for the exponential of a skew-symmetric matrix:

$$
\exp (\tilde{\theta})=\mathbf{I}+\tilde{u} \sin \left(\|\theta\|_{2}\right)+\tilde{u}^{2}\left\{1-\cos \left(\|\theta\|_{2}\right)\right\}
$$

This result employs only a scalar power series and therefore it avoids the numerical difficulties of matrix exponentiation [24].

\section{RESULTS AND DISCUSSION}

The model and solution algorikm described above is now used to explore several possible dynamic motions that are generated by buckling an example cable by twisting one end. The model parameters that define the example are listed in Table 1 and Table 2 and a schematic of the model is illustrated in Fig. 5.

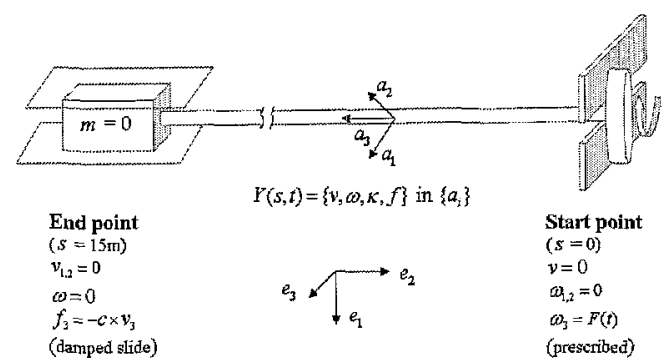

Figure 5: A low tension cable under increasing twist created by rotating the right end. Left end may be free to slide or have prescribed sliding velocity and/or reaction (tension).

${ }^{3}$ Note that Eq. (2) follows from taking the time-derivative of Eq. (25) and equating it to the spatial derivative of Eq. (23). Use the fact that $\tilde{\boldsymbol{K}} \tilde{\omega}-\tilde{\omega} \tilde{\boldsymbol{K}}$ is the skew-symmetric form of $\kappa \times \omega$.

${ }^{4}$ Substitute $\tilde{u}^{3}=-\tilde{u}$ in Taylor expansion to verify this formula.
Table 1: Cable and simulation parameters.

\begin{tabular}{|c|c|c|}
\hline Quantity & Units $($ SI $)$ & Value \\
\hline Young's Modulus, $E$ & $\mathrm{~Pa}$ & $2.0 \times 10^{7}$ \\
\hline Shear Modulus, $G$ & $\mathrm{~Pa}$ & $3.5 \times 10^{6}$ \\
\hline Cable Diameter, $D$ & $\mathrm{~m}$ & $1.7 \times 10^{-2}$ \\
\hline Cable Length, $L$ & $\mathrm{~m}$ & $1.5 \times 10^{1}$ \\
\hline Cable Density, $\rho_{c}$ & $\mathrm{Kg} / \mathrm{m}^{3}$ & $4.0 \times 10^{2}$ \\
\hline Fluid Density, $\rho_{w}$ & $\mathrm{Kg} / \mathrm{m}^{3}$ & $1.0 \times 10^{0}$ \\
\hline Drag Coefficient ${ }^{5}, C_{d}$ & - & $3.0 \times 10^{1}$ \\
\hline Temporal Step, $\Delta t$ & $\mathrm{~s}$ & $2.0 \times 10^{-1}$ \\
\hline Spatial Step, $\Delta s$ & $\mathrm{~m}$ & $1.0 \times 10^{-1}$ \\
\hline
\end{tabular}

Table 2: Cable cross-section properties.

\begin{tabular}{|c|c|c|}
\hline Quantity & Formula & Units (SI) \\
\hline Cross-section Area & $A_{c}=\frac{\pi D^{2}}{4}$ & $\mathrm{~m}^{2}$ \\
\hline Area Moments of Inertia (bending) & $J_{1,2}=\frac{A D^{2}}{16}$ & $\mathrm{~m}^{4}$ \\
\hline Area Moments of Inertia (torsion) & $J_{3}=\frac{A_{c} D^{2}}{8}$ & $\mathrm{~m}^{4}$ \\
\hline $\begin{array}{c}\text { Mass Moments of Inertia per unit } \\
\text { cable length (bending) }\end{array}$ & $I_{1,2}=p_{c} I_{t, 2}$ & $\mathrm{Kg}-\mathrm{m}$ \\
\hline $\begin{array}{c}\text { Mass Moments of Inertia per unit } \\
\text { cable length (torsion) }\end{array}$ & $I_{3}=p_{c} J_{3}$ & $\mathrm{Kg}-\mathrm{m}$ \\
\hline
\end{tabular}

From Fig. 5, we infer that the right end (referred to as the "Start point", $s=0$ ) of the cable is subjected to a slowly increasing rotation about the $a_{3}$ axis. This end cannot move and is otherwise constrained in rotation (no rotation about the $a_{1}$ and $a_{2}$ axes). The left end (referred to as the "End point", $s=L$ ) of the cable is fully restrained in rotation (about all three axes) and also cannot translate in the transverse $\left(a_{1}-a_{2}\right)$ plane. This end, however, may slide horizontally along the inertial $e_{2}$ axis and this translation may also be partially restrained by added damping (damping parameter $c$ ). This added damping will be later used to control the time scale of the dynamic response including any eventual collapse of the cable.

The integration begins with the cable initially horizontal and stress-free. It is in a fluid (with no flow) that provides both drag and added mass (as modeled by a Morison formulation). We also assume no intrinsic curvature, structural damping, or gravity. However, we do add a minute initial distributed force in the upward $\left(-e_{1}\right)$ direction to initiate a subsequent buckling.

A first problem is considered in which the right end of the cable is subjected to increasing rotation about the loading $\left(e_{2}\right)$ axis. This is achieved by prescribing the angular velocity component $\omega_{3}$ as illustrated in Fig. 6. In this example, the angular velocity is increased

\footnotetext{
${ }^{5}$ Distributed Drag $=\frac{1}{2} C_{d} \rho_{w} S \quad \times\left(\right.$ relative flow velocity ${ }^{2}$, where $S=D$ for normal drag and $S=\pi D$ for tangential drag (skin friction) [18]. We increased $C_{d}$ 2-fold during the dynamic collapse (from the cable state in Fig. (8) to the state in Fig. (9)). This helped slow down the otherwise drastic dynamics that accompany the sudden collapse.
} 

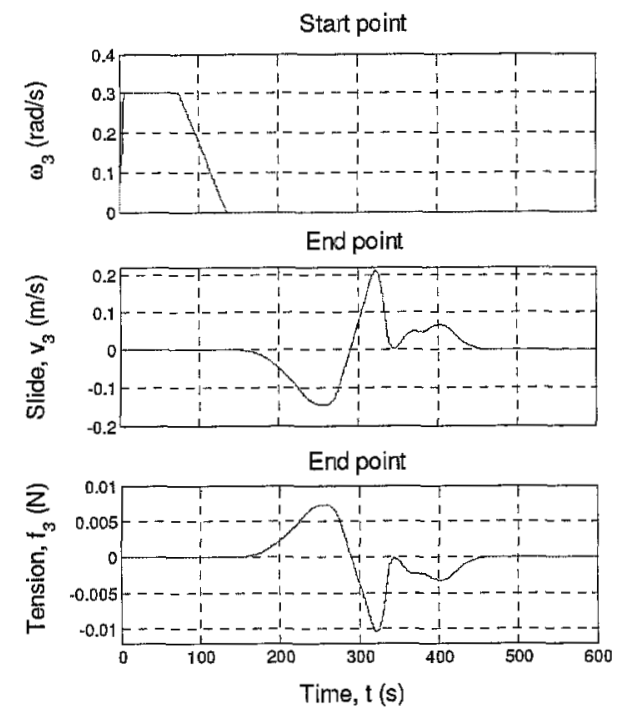

Figure 6: Boundary conditions. The starting point (right end) is subjected to a prescribed angular speed about the tangent. The end point (left encl) is free to slide under linear viscous damping.

from zero to a constant value $(0.3 \mathrm{rad} / \mathrm{sec})$ and then slowly reduced until it reaches zero and held at zero thereafter. The resulting twist that is induced in the cable is sufficient to generate torsional buckling and subsequent dynamic response. The dynamic response is influenced by the motion of the left end as illustrated in Fig. 6. In particular, the left end is held fixed during the period when the cable is being twisted (first $135 \mathrm{sec}$ ). After this period, the left end is allowed to slide towards the right end under the influence of a viscous reaction force (creating end tension, $f_{3}(L, t)$ proportional to the sliding velocity). The dynamic deformation that results from these boundary conditions is discussed next.

As the right end is first twisted by a modest amount, the cable remains straight. There is an abrupt change however when the twist reaches a critical value (at approximately $145 \mathrm{sec}$ ) when the Greenhill buckling condition [25] is achieved and the strajght (trivial) configuration becomes unstable. The model employed here captures this initial instability as well as the subsequent nonlinear deformations that generate the post-buckled geometry of the cable. The geometry just after initial buckling is approximately helical as can be observed in Fig. 7, which provides a snap-shot at 200 seconds. Shown are projections of the three-dimensional cable geometry in the three principal planes of the inertial frame. An isometric view is also included. Notice that the cable appears to make a single helical turn that also lies wholly above the horizontal $\left(e_{2}-e_{3}\right)$ plane as expected from the first buckling mode of the (simpler) linearized theory [25].

As the left end is allowed to slide towards the right end, the helical cable undergoes a secondary buckling in which it suddenly collapses in forming a (nearly) planar loop with self-contact. This collapse occurs at approximately $280 \mathrm{sec}$. in this example. The dynamic collapse is predicted from investigations of the stability of
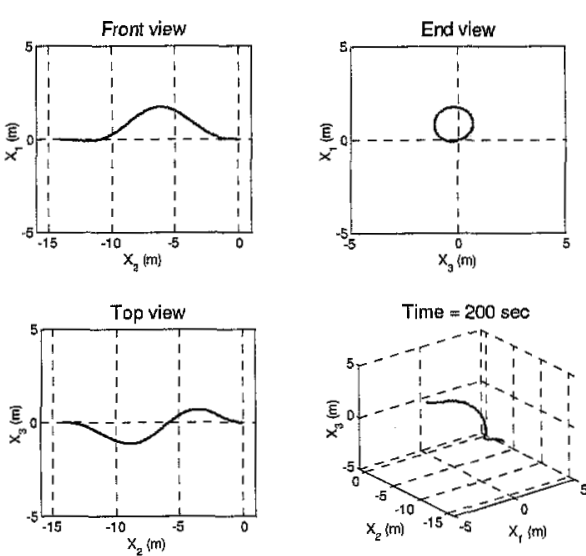

Figure 7: Approximately helical shape (or toroidal writhe) following initial torsional buckling.

the equilibrium forms of a cable/rod under similar loading conditions; refer to Lu and Perkins [6] and studies cited therein. These results here extend to the dynamic regime of these studies that previously focused on equilibrium alone. Figure 8 shows a snap-shot of the three-dimensional shape of the cable just before dynamic collapse (at $255 \mathrm{sec}$ ). Note that the center of the cable has rotated a total of $90^{\circ}$ about the vertical $\left(e_{1}\right)$ axis so that the tangent at this (mid-span) point is now orthogonal to the loading $\left(e_{2}\right)$ axis. This was a noted bifurcation condition in Lu and Perkins [6] at which the equilibrium form loses stability. The dynamic collapse thereafter is depicted in Fig. 9, which now illustrates the cable a short time later $(281 \mathrm{sec})$. This cable now is nearly planar and forms a closed-loop.

The present formulation, however, ignores cable self-contact and therefore the loop shown in Fig. 9 is only temporary. Contact forces would be required to stabilize this loop. Instead, our model allows the cable to pass through itself as the momentum of the collapse carries the cable through this looped configuration and towards a stable configuration in the shape of straight but twisted cable. During this final process, the twist is reduced and is finally insufficient to initiate further torsional buckling (i.e., below the Greenhill condition).

The entire dynamic collapse depicted by the sequence shown in Figs. 7-9 involves a conversion of torsional strain energy to bending strain energy. The analogous process exhibited by DNA strands involves the dynamic conversion of twist to writhe and it accompanies the transition of DNA from one supercoiled state to another [9]. Prior analyses of these conversions have relied on equilibrium (static) models of DNA [10-17] and truly dynamic transitions, such as those in Fig. 7, Fig. 8 and Fig. 9 are poorly understood [9]. We endeavor to provide some understanding of this dynamic process by referring to Fig. 10 . 

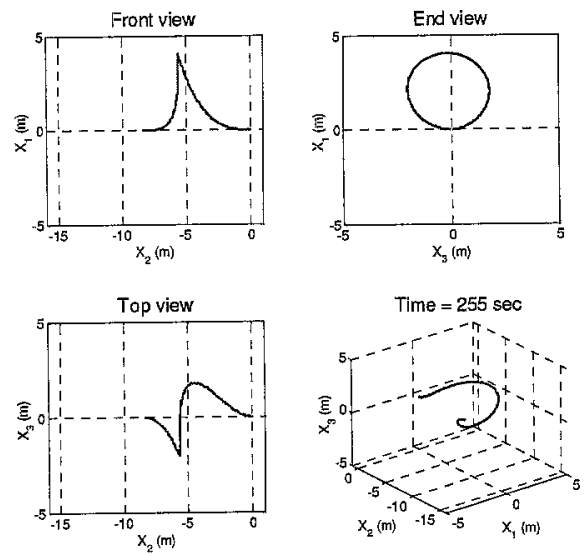

Figure 8: Just prior to collapse, the tangent $a_{3}$ at the midpoint becomes perpendicular to the loading axis $e_{2}$.

We start with the result of Calugareanu [26] and White [27] for closed loops (for example, DNA plasmid) that states that the linking number $L k$ is conserved as is equal to the sum of total twist $T w$ and writhe $W r$

$$
L k=T w+W r
$$

The linking number $L k$ represents the number of turns included in the closed cable loop as shown in Fig. 12. These turns are considered positive or negative based on whether they are right-handed or left-handed. It is intuitive that we can't alter the linking number without cutting through a section of the cable. If the centerline curve of the loop cable is planar (and non-intersecting, i.e. the cable has non-zero thickness), all the turns are in the form of pure twist and thus the number of turns equals to the total twist $T w$. In Fig. 11, this number can be counted as the number of black regions in the circular loop. The twist can be released and doing so creates writhe as observed in the succession of loops shown in Fig. 11. The writhe $W r$ is purely a function of the space curve and quantifies the number of cross-overs one can see averaged over all possible views. For a detailed discussion, refer to Calladine and Drew [9] and for computation of the writhe $\mathrm{Wr}$ refer to Fuller [28].

In open structures such as ours (length of cable or DNA strand), this theorem still applies provided no further rotations are allowed at the boundaries (as in the boundary conditions for this example) and the structure is not "cut". This is illustrated in Fig. 12. We compute the total twist $T w$ from

$$
T w=\frac{1}{2 \pi} \int_{0}^{L} \kappa_{3} d s
$$
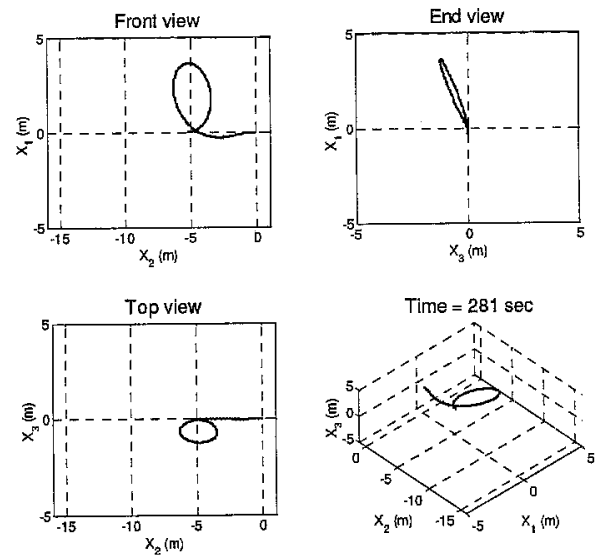

Figure 9: Loop formation. The cable of Fig. 8 collapses into a planar loop shortly thereafter.
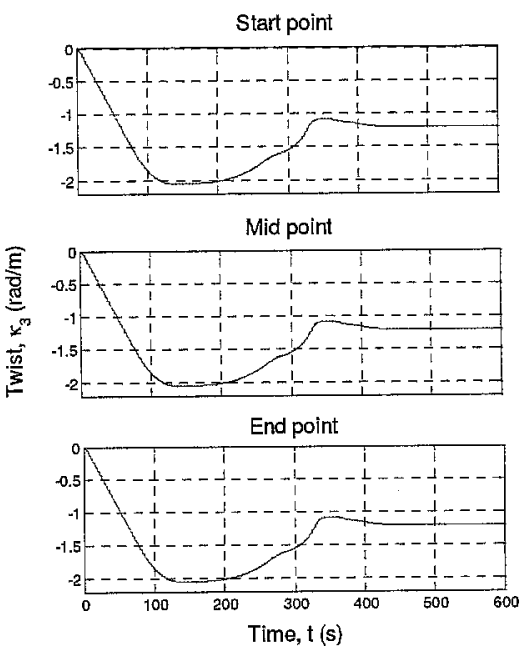

Figure 10: Loss of twist during buckling due to released torsional strain energy.

and refer to the reviews by Tobias et al. [10] and Coleman et al. [11] for computing the writhe $W r$ in open structures.

In our example, the initial twisting phase (up to $135 \mathrm{sec}$ ) introduces approximately $4.8 \mathrm{Lk}$, all in the form of twist, prior to the initial buckling (see Fig. 13). This link is conserved just prior to the would-be self-contact during the collapse, as the ends of the cable are prevented from further rotation. During the initial buckling and secondary collapse, the cable exchanges $T w$ for $W r$ by transferring torsional strain energy into bending strain energy. For instance, just 


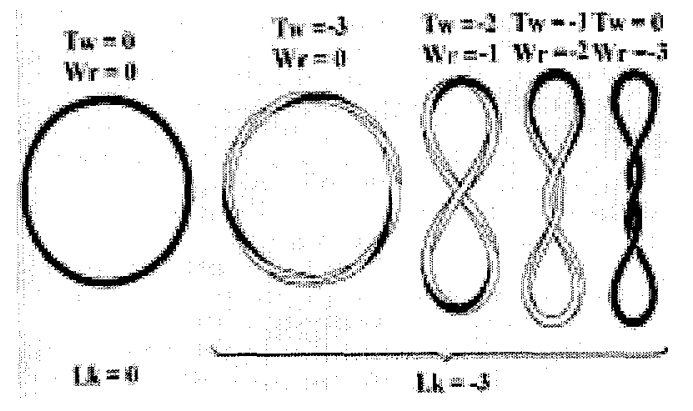

Figure 11: The linking number in the left-most cable loop is 0 and in the next loop it is -3 . The twist is converted to successively greater writhe in the remaining loops. (Illustrations courtesy of [9]).

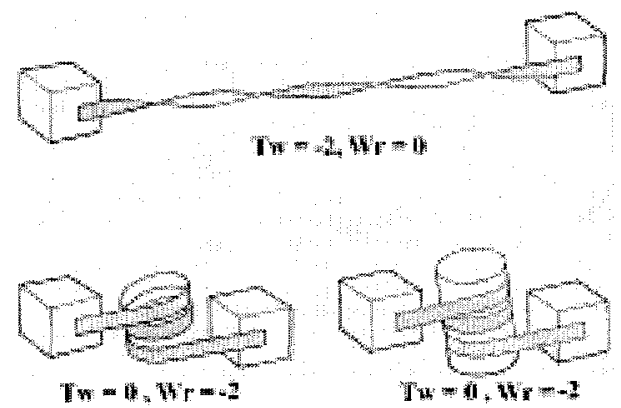

Figure 12: The end blocks do not rotate and only translate towards each other. These end conditions conserve the linking number. Twist in the top strand converts to writhe. (Illustrations courtesy of [9]).

before the "self-contact", the cable has $1.0 \mathrm{Wr}$ and $3.8 \mathrm{Tw}$. Just after "self-contact" our model allows the cable to pass through itself and in doing so $L k$ is (rather artificially) reduced by 2.0 . Also, just after passing through "contact", Wr becomes -1.0 (changes sign). Beyond this point, the cable further releases both bending and strain energy in settling to a (stable) straight equilibrium with pure twist (and $2.8 \mathrm{Lk}$ ). So in this final phase, one $T w$ annihilates one $W r$ in arriving at a twisted but straight cable. This dynamic process is captured in the results of Fig. 13 .

\section{SUMMARY AND CONCLUSIONS}

This paper reviews a rod theory and a numerical algorithm that can be used to study the nonlinear dynamics of highly contorted cables. While the primary objective is to model the dynamics of marine cables leading to the formation of loops and tangles on the sea floor, it is also recognized that the same techniques may apply to modeling the supercoiled states of DNA and the dynamic transitions between these states. These techniques are used herein to study the response of a prototypical system, composed of an elastic cable subjected to increasing twist. Numerical simulations reveal that the originally straight cable undergoes two bifurcations in succession as

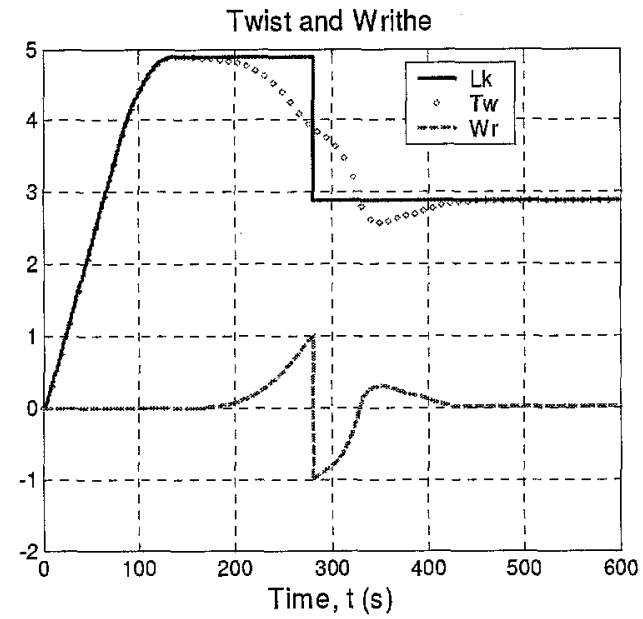

Figure 13: Variation of twist and writhe. The discontinuous fall in the linking number and correspondingly in the writhe occurs when the cable passes through itself.

twist is added. The first of occurs at the Greenhill buckling condition where the trivial (straight) equilibrium becomes unstable and the cable buckles into the approximate shape of a helix. This helix grows in amplitude with increasing twist. One measure of this growth is the continued rotation of the tangent at the mid-span point. When this tangent becomes orthogonal to the loading axis (axis of the original straight cable), the helix experiences a second bifurcation and collapses dynamically towards a loop. The current model, which does not model self-contact, cannot capture the stable loop that is expected to form when self-contact is included. The addition of selfcontact is a current topic of research.

ACKNOWLEDGMENTS: The authors gratefully acknowledge the research support provided by the U.S. Office of Naval Research and the Lawrence Livermore National Laboratories. The work of Christopher L. Lee was performed under the auspices of the U.S. Department of Energy by the University of California, Lawrence Livermore National Laboratory under contract No. W-7405-ENG-48.

\section{REFERENCES}

[1] Coyne J., 1990, "Analysis of the Formation and Elimination of Loops in Twisted Cable", IEEE Journal of Oceanic Engineering, 15, pp. $72-83$.

[2] Rosenthal F., 1976, "The Application of Greenhill's Formula to Cable Hockling", Journal of Applied Mechanics, 43, pp. 681-683.

[3] Rosenthal F., 1975, "Greenhill's Formula and the Mechanics of Cable Hockling", NRL Report 7940, Naval Research Laboratory, Washington D.C.

[4] Liu F. C., 1975, "Kink Formation and Rotational Response of Single and Multistrand Electromechanical Cables", Technical Note $\mathrm{N}-1403$, Civil engineering Laboratory, Naval Construction Batallion Center, Port Hueneme, CA

[5] Tan Z. and Witz J. A., 1993, "On the Flexural-Torsional Behavior of a Straight Elastic Bean Subject to Terminal Moments", Journal of Applied Mechanics, 60, pp. 498-505. 
[6] Lu C.L. and Perkins N.C., 1995, "Complex Spatial Equilibria of U-joint Supported Cables under Torque, Thrust and Self-weight", International Journal of Non-linear Mechanics, 30, pp. 271-285.

[7] Lu C.L. and Perkins N.C., 1994, "Nonlinear Spatial Equilibria and Stability of Cables under Uni-axial Torque and Thrust", Journal of Applied Mechanics, 61, pp. 879-886.

[8] Gatti-Bono C. and Perkins N. C., 2002, "Dynamic Analysis of Loop Formation in Cables under Compression", International Journal of Offshore and Polar Engineering, 12, pp. 217-222.

[9] Calladine C. R. and Drew H. R., 1997, Understanding DNA, the molecule and how it works, Academic Press, chapter 6, pp. 120144.

[10] Tobias I., Swigon D. and Coleman B. D., 2000, "Elastic Stability of DNA Configurations. I. General Theory", Physical Review E, 61, pp. 747-758.

[11] Coleman B. D, Swigon D. and Tobias I., 2000, "Elastic Stability of DNA Configurations. II. Supercoiled Plasmids with Self-contact", Physical Review E, 61, pp. 759-770.

[12] Gonzales O., Maddocks J. H., Schuricht F, and von der Mosel H., 2002, "Global Curvature and Self-contact of Nonlinearly Elastic Curves", Calculus of Variations and Partial Differential Equations, 14, pp. 29-68.

[13] Manning R. S., Maddocks J. H. and Kahn J.D., 1996, "A Continuum Rod Model of Sequence-dependent DNA Structure", Journal of Chemical Physics, 105, pp. 5626-5646.

[14] Schlick T. and Ramachandranan G., 1997, "Buckling transitions in Superhelical DNA: Dependence on the Elastic Constants and DNA size", Biopolymers, 41, pp. 5-25.

[15] Schlick T., 1995, "Modeling Superhelical DNA: Recent Analytical and Dynamic Approaches", Current Opinion in Structural Biology, 5, pp. 245-262.

[16] Olson W. K. and Zhurkin V. B., 2000, "Modeling DNA deformations", Current Opinion in Structural Biology, 10, pp. 286297.

[17] Fain B., Rudnick J. and Ostlund S., 1997, "Conformations of linear DNA", Physical Review E, 55, pp. 7364-7368.

[18] Gatti-Bono C. and Perkins N. C., 2002, "Physical and Numerical Modeling of the Dynamic Behavior of a Fly Line", Journal of Sound and Vibration, 225, pp. 555-577.

[19] Gobat J. I. and Grosenbaugh M. A., 2001, "Application of the Generalized-Alpha Method to the Time Integration of the Cable Dynamics Equations", Computational Methods in Applied Mechanics and Engineering, 190, pp. 4817-4829.

[20] Chung J. and Hulbert G. M., 1993, "A time Integration Algorithm for Structural Dynamics With Improved Numerical Dissipation - The Generalized-Alpha Method", Journal of Applied Mechanics, 60, pp. 371-375.

[21] E. H. Love, 1927, A Treatise on the Mathematical Theory of Elasticity, Dover Publications, New York, Chapter 18, pp. 281-284.

[22] Gobat J. I., Grosenbaugh M. A and Triantofyllou M. S., 2002, "Generalized-Alpha Time Integration Solutions for Hanging Chain Dynamics", Journal of Engineering Mechanics, 128, pp. 677-687.

[23] Sun Y., 1996, "Modeling and Simulation of Low-tension Oceanic Cable/ Body Deployment, Ph.D. Dissertation, University of Connecticut.

[24] Moler C. and Van-Loan C., 1978, "19 Dubious Ways to Compute Exponential of a Matrix", SIAM Review, 20, pp. 801-836.

[25] Timoshenko S. P. and Gere J. M., 1961, Theory of Elastic Stability, McGraw-Hill Book Company, New York, Chapter 2, pp. 156-157.
[26] Calugareanu G., 1961, Czechoslovak Math. J., 11, pp. 588 [27] White J. H., 1969, "Self-Linking and Gauss-Integral in Higher Dimensions", American Journal of Mathematics, 91, pp. 693.

[28] Fuller F. B., 1971, "Writhing Number of a Space Curve", Proceedings of the National Academy of Sciences of the United States of America, 68, pp. 815.

[29] Nelson D. L. and Cox M. M., 2000, Lehninger Principles of Biochemistry, Worth Publishers, chapter 24, pp. 915-930. 